\title{
THE STATE OF PROTOTYPING PRACTICE IN THE INDUSTRIAL SETTING: POTENTIAL, CHALLENGES AND IMPLICATIONS
}

\author{
Diefenbach, Sarah (1); Christoforakos, Lara (1); Maisch, Bettina (2); Kohler, Kirstin (3) \\ 1: Ludwig-Maximilians-University Munich; 2: Siemens AG, Munich; 3: University of Applied Sciences \\ Mannheim
}

\begin{abstract}
Prototyping as a central method within innovation- and product development processes has a high acceptance in industry. Various prototyping tools provide impressive visualizations of product ideas in early development stages and especially low fidelity prototyping methods seem easily applicable. However, a closer look at prototyping in practice reveals a number of misunderstandings and barriers regarding effective prototyping, often related to different stakeholders' (e.g., developer, designer, client) ideas about the purpose of prototyping. Based on a combination of literature analysis, adapting existing models and methods in user centered design (e.g., personas, double diamond design process) and empirical results from industrial research cooperation, we introduce a focus group format and a first model of prototyping maturity, which can help organizations to reflect on their state of practice in prototyping on an individual, team, and organizational level. The maturity model also forms a valuable theoretical lens for design research. Thus, our research aims at researchers in the field of prototyping as well as practitioners involved in prototyping and innovation processes.
\end{abstract}

Keywords: Design process, User centred design, Design practice, Prototyping, Innovation methods

\section{Contact:}

Diefenbach, Sarah

Ludwig-Maximilians-University Munich

Psychologie

Germany

sarah.diefenbach@1mu.de

Cite this article: Diefenbach, S., Christoforakos, L., Maisch, B., Kohler, K. (2019) 'The State of Prototyping Practice in the Industrial Setting: Potential, Challenges and Implications', in Proceedings of the 22nd International Conference on Engineering Design (ICED19), Delft, The Netherlands, 5-8 August 2019. DOI:10.1017/dsi.2019.176 


\section{INTRODUCTION}

Prototyping, as a central method of innovation and development processes has a high acceptance in industry. However, while introducing it to organizations might seem easy, the industrial practice reveals several misunderstandings and unsatisfying outcomes regarding the effective use of prototypes. For example, different stakeholders hold different expectations from prototyping, and thus, have different preferences for methods or tools. While engineers primarily prototype to insure technical feasibility, designers at the fuzzy frontend (e.g. early phases of design thinking projects) primarily prototype to communicate and identify user needs, to name one typical misunderstanding. Based on a research cooperation with Siemens, in this paper we introduce a focus group format that can help to systematically assess barriers of stakeholders with respect to prototyping. The results illustrate typical shortcomings and barriers of prototyping practice in industrial settings, which we have also experienced in previous projects. To support a systematic identification of weaknesses and consideration of appropriate actions, we propose a maturity model to help organizations identify and overcome current shortcomings within prototyping. It might also form a valuable theoretical lens for prototyping research. Hence, the paper is targeted at researchers in the field of prototyping and practitioners responsible for innovation processes within large and medium sized organizations. Our work is limited to the domain of digital innovation projects, including service design with digital parts to support the software, pure software products and smart objects, i.e. products with digital elements.

\section{THEORY AND CHALLENGES OF PROTOTYPING}

To lay a common ground for this paper in terms to terminology, we start with a clarification of terms and definitions. We further elaborate on different perspectives regarding the systematization of prototypes as described in the literature building our work's foundation. Our understanding of prototyping parallels that of Lim et al. (2008), who introduced the "Anatomy of Prototypes". Accordingly, prototypes consist of the dimensions "filter" and "manifestation". Thus, they filter important aspects of a design idea and manifest those in a tangible prototype. This broad definition of prototyping does not restrict the manifestation to a specific material. Any kind of manifestation of the design idea, like a simple roleplay, can be considered a prototype. Also note that the present discussion focuses on prototyping in the sense of conceptualization, namely, the exploration, communication and evaluation of product concepts, and not prototyping from a technical perspective.

\subsection{Purpose of prototyping}

A prototype can be used for exploration, communication and/or evaluation (Blomkvist \& Holmlid, 2011; Houde \& Hill, 1997). Although prominent literature repeatedly underlines the importance of the fit between a prototyping method or tool and its underlying research question and purpose (e.g. Schneider, 1996), studies show that practitioners often lack reflection and structure in the choice and implementation of prototypes. Instead, they often rely on organizational routines (e.g. Christoforakos \& Diefenbach, 2017; Diefenbach et al., 2013), leading to prototypes not fulfilling their purpose with respect to initial research questions. Research literature offers several perspectives that are helpful to identify the appropriate prototyping approach for a given context and prototyping purpose. For example, the purpose to a prototype could be categorized in relation to different stakeholders' questions and responsibilities (as elaborated in 2.2), but also the project phase (as elaborated in 2.3). Ideally these perspectives should be aligned when choosing an appropriate prototyping approach.

\subsection{A Stakeholder-based perspective}

The target group has been repeatedly identified as a central aspect for the choice of appropriate prototyping methods. Blomkvist and Holmlid (2011) categorize such in clients, users and colleagues. They point out that due to different responsibilities, theses stakeholders follow different purposes in prototyping. Based on previous research and focus groups within different organizations (Christoforakos et al., 2018), we propose an initial set of stakeholder categories in product design, including, for example, interaction designer, project lead, and client. Based on our UX/HCD focus, we look at prototypes from a stakeholder perspective, considering that such are applied within a particular context as well as in interaction with a specific target group. Thus, each stakeholder and the 
corresponding purpose of prototyping in different design phases can affect the prototyping method and fidelity. These exemplifying stakeholders are illustrated in the form of personas (e.g. Chang et al., 2008; Pruitt \& Grudin, 2003), as fictional characters with typical demographic attributes, goals, motivations and behavior patterns as guidance for choosing methods based on such stakeholder's needs and project context.

\subsection{A Process-based perspective: The double diamond}

The double diamond published by the Design Council (2007) serves as a model to describe the phases of the design process. It consists of four phases with different impacts on the usage of prototypes. It builds upon the assumption that the design of a product is manifested in a design space. This includes all knowledge about the problem and possible solutions. As such, the design space can be separated in the problem space and the solution space, each of them defining one diamond in the model. Furthermore, the model distinguishes divergent and convergent activities. Divergent activities open the design space by gaining knowledge and pointing out alternatives. Convergent activities like synthesis and decision-making narrow it. The double diamond, as shown in Figure 1, distinguishes the following four phases:

- Discover: During this phase, knowledge and insights about the user are generated and the context and stakeholders are collected. Prototypes are built to learn about the user and the context.

- Define: During this phase, gained knowledge is filtered and analyzed. At the end the design opportunity is defined. In design thinking, this is also known as "Point of View". It is an important point in the process of design innovation, as at that point companies often decide about the project's further direction. Prototypes are built to validate assumptions and compare alternative ideas in terms of feasibility and user experience as well as for communication to decision makers.

- Develop: In this phase, solutions for the design opportunity are explored. The design is further refined. Prototypes are used to explore and evaluate design decisions within a defined solution space referring to the design opportunity. Subsequent prototypes explore and validate the solution.

- Deliver: The last phase brings the product to the market. In this phase, evolutionary prototypes are built for evaluation.

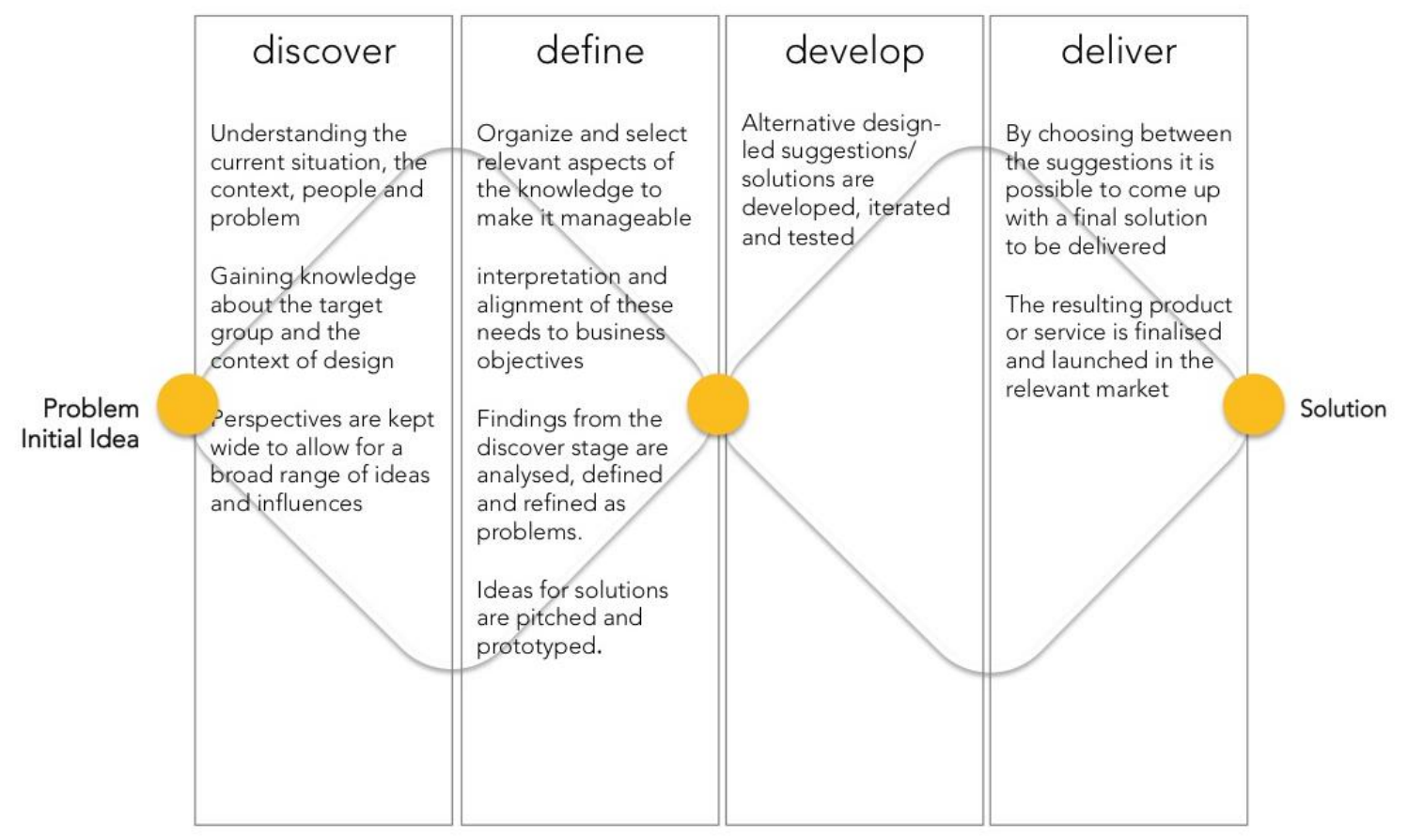

Figure 1. Illustration of the four phases of the double diamond model (2007) annotated with the emphasis of these phases in respect to prototyping 


\section{A FOCUS GROUP AS A STARTING POINT TO ENHANCE A COMPANY'S PROTOTYPING READINESS - A CASE STUDY AT SIEMENS}

A focus group at Siemens was conducted to investigate the state of practice regarding prototyping at various departments. Qualitative data about potential areas of improvement was collected and a systematization of prototyping through perspectives on it was introduced. The aim was to further support processes of reflection regarding prototyping within Siemens practitioners. The following sections elaborate on the focus group and the according findings.

\subsection{Method: Conducting the focus group}

Fifteen stakeholders from various departments were invited. The workshop was announced in the internal social network platform. It was communicated that it targeted at stakeholders within innovation processes for digital products; creating, evaluating or presenting prototypes, namely: Product Owners, R\&D Managers, Development Engineers, Innovation Leads, UX Designers, Interaction Designers. All participants belonged to different business units and were thus responsible for varying digital products.

Participants were asked to prepare prototyping experiences and share a typical prototype they interacted with. The workshop lasted 4,5 hours and included the following phases:

- Introduction of moderators and participants: After an introduction of the moderators, participants were requested to elaborate on their prototyping experience by a short summary of a special prototype. This was supposed to create awareness of current challenges and enabled referring to such, when moderators provided theoretical background at later stages.

- Introduction of basic terms: As prototyping can have different meanings and purposes depending on stakeholders and contexts of application, it seems impossible to sufficiently enumerate methods and their definitions. In accordance, stakeholders of different backgrounds and positions participated at the workshop, all bringing their own understanding of prototyping. To enhance communication and allow participants to express experiences and pitfalls without misunderstandings, a basic prototyping vocabulary was established, including the understanding of prototypes as filters and terms like fidelity, materials and tools for prototyping.

- Stakeholder perspective: The initial personas were introduced. Likewise, purposes of a prototype (ideation, communication, evaluation) and how it might interfere with the stakeholder role was presented. In subgroups participants stated which persona best represents their role with the flexibility of adjusting descriptions if necessary, as well as for which purpose they prototype.

- Method/artefact perspective: Furthermore, various methods ranging from textual prototyping and mock-ups to methods like invisible design were presented with the purpose of raising awareness regarding the large variety of materials and fidelity of prototypes, as well as their relationship to the method used to create or validate it, highlighting, that the choice of the appropriate method has a huge impact on the outcome and the efficiency of the prototyping effort. Several diverse examples were demonstrated, which pointed to a deliberate selection of the method that fits the context of the project. In addition, we described challenges of prototyping as described in the literature to inspire the reflection process. Based on this input, participants were requested to refer to their prototyping story from the workshop's beginning and judge how well their choice of material, method and purpose of prototype was, as well as whether they observed problems or inefficiencies in prototyping that could have been caused by not being aware of the described dependencies.

- Design process perspective: A theoretical introduction into the double diamond process was given and participants were asked to reflect in which phase their project was, as well as to place their prototypes into the different phases and judge, whether their prototype's focus fitted the phase. This reflection was guided with cards, stating the purpose and type of prototype for each phase.

- Closing: After these intense phases of working in subgroups, the workshop was closed by a summary of each participant about what he/she took out of the workshop. Participants were also asked to state their wishes regarding the organization to improve their daily work with prototypes. 


\subsection{Findings}

\subsubsection{Prototyping practice at Siemens - Current status}

Participants' reflections on prototyping practices at Siemens revealed insights of how prototyping is currently used and understood, as well as typical problems and challenges.

Prototypes fulfill different purposes related to different phases of product development. For example, prototypes are first used in a creative way, to generate ideas. Later, they are used to present proposals and to document already taken decisions (e.g., about product features). Finally, prototypes are also used for evaluation, i.e., getting quick feedback, tackling risks, and thus validating product concepts at early development stages. In fact, a central benefit of prototyping is to "fail early" and to rethink design decisions before cost-intensive implementations are performed. Ironically, several participants mentioned that no (or less than necessary) prototypes were built due to limited project budget.

Prototypes have different forms, ranging from paper and other prototypes of low-cost material, Lego prototypes, 3D prints, low-tech physical prototypes or prototypes created by laser cutting up to hightech prototypes integrated in the software of productive systems. The participants' prototypes referred to very different aspects of the product (in Lim's term they represented different "Filters"): Lego prototypes for example served as representations of a service concept the digital product might support, laser cutting or 3D prints represented physical manifestations of smart-objects.

In line with the stakeholder-based categorization of prototyping, workshop participants also mentioned different prototyping purposes related to different groups of persons: From an internal perspective, prototypes can for example help to avoid misunderstandings within the development team, and to get a better and shared understanding of the project across teams. It was emphasized that prototypes are a valid opportunity to bring everybody up to the same level ("an image is worth a thousand words"). From an external perspective, prototypes provide a valid basis to engage and communicate with the customer. The summarized purposes mirror a very general, traditional perspective on prototyping, which seems to be relevant within the organization, but leave out further essential purposes including exploration/ideation and evaluation.

In general, personas revealed that in a large company like Siemens, a lot of people are engaged in prototyping. For example, participants added "product owner" and "intrapreneur" as additional stakeholders. However, these stakeholders' roles and relations to prototyping is seldom explicitly communicated and not mirrored in development processes. Thus, it needed this explicit reflection on prototyping stakeholders to sensitize people for the various perspectives on prototyping within a company and the need to consider these in applied prototyping methods.

Besides the emphasis on current benefits through prototyping, participants also mentioned many existing challenges in prototyping in an effective and efficient way. One cluster of statements related to inefficiency based on a lack of communication between different persons engaged in the development process, often resulting in double work. For example, participants noted that different departments typically used different prototyping software and rebuilt the same prototype using another technical realization, thus not gaining additional insights. Confusion and misunderstandings as a result of lacking communication about prototyping on a meta-level also became obvious in participants' positioning of prototypes in the different double diamond phases (as described under process perspective). Note, however, that depending on the project, some phases of the double diamond might be less relevant or not applicable at all. Some teams might directly start their projects in the solution space. In some cases, participants' positioning of the same prototype differed considerably. Besides different interpretations of the double diamond model, this might imply a missing shared understanding of why and for which insights a particular prototype is used.

Another problem was the strong focus on technical features and details but a lack of emphasis on experiential aspects and the product concept from a holistic perspective. This was, for example, expressed in statements such as "the holistic picture is missing in all the teams/departments involved in the same process", "many departments focus on technical prototypes only", "prototypes too often focus on detailed features", "no experience prototypes", "specification of user experience and user flow are not prioritized" or" "stakeholders are more interested in function than user experience"”. Related to the missing emphasis on UX during a large part of the development process, participants also reported of difficulties in expectation management and frequent disappointment due to gaps between prototypes and final products. This was often related to the experience that in the course of 
product development and different prototypes, the original concept was not successfully transferred and "got lost". One participant described this as the "success of prototype over concept", often resulting in the final product not fulfilling the original user requirements and thus not being used. Finally, another cluster of statements referred to ineffective prototyping due to missing knowledge, skills or resources. This could be simple barriers such as people being reluctant to draw (which prevents the creation of early concept sketches and low fidelity prototyping), missing knowledge on how to find the appropriate type of prototype for different purposes or how to identify and test the most critical assumption, or missing contact/access to the end users for realistic concept evaluation.

\subsubsection{Analysis and recommendations}

For a closer insight to potential sources of the identified barriers to efficient prototyping, we clustered identified topics and categorized them into barriers on different levels. Broadly, the barriers can be differentiated into barriers on the individual level, such as lack of individual knowledge or awareness of challenges within prototyping, barriers on a team level, such as lacking communication between internal prototyping stakeholders or lacking standards resulting in double work, and barriers on the organizational level, such as lacking appreciation and resources for prototyping or lacking access to relevant target groups. Note, however, that the categorization is not always clear cut, since barriers on a higher level typically also affect lower levels (e.g., a lacking integration of prototyping in development processes on an organizational level promotes lacking communication about prototyping on a team level). Table 1 gives an overview of the identified barriers on the different levels.

Table 1. Barriers to successful prototyping at Siemens on different levels

\begin{tabular}{|c|c|c|}
\hline Level & Barrier & Examples \\
\hline Individual & $\begin{array}{l}\text { lacking awareness of } \\
\text { suitability of methods for } \\
\text { different purposes }\end{array}$ & $\begin{array}{l}\text { lacking awareness of positioning in double diamond } \\
\text { and matching particular prototyping methods to } \\
\text { stakeholders }\end{array}$ \\
\hline Individual & $\begin{array}{l}\text { lacking awareness of full } \\
\text { spectrum of prototyping } \\
\text { methods }\end{array}$ & $\begin{array}{l}\text { too narrow spectrum of "prototypes" in use, e.g., focus } \\
\text { on technologically advanced prototypes and testing of } \\
\text { functions from a technical perspective, disregard of } \\
\text { low fidelity methods and exploration of functions from } \\
\text { a UX/experiential perspective }\end{array}$ \\
\hline Individual & $\begin{array}{l}\text { lacking awareness of risks } \\
\text { and biases related to use of } \\
\text { prototypes for evaluation }\end{array}$ & $\begin{array}{l}\text { lacking awareness for idealization tendencies and need } \\
\text { for iterative prototypes and evaluation studies, e.g., } \\
\text { "we have implemented all the stuff the users wished } \\
\text { for - nevertheless they don't like the final product" }\end{array}$ \\
\hline Team & lacking standards & $\begin{array}{l}\text { no prototyping standards, many different tools, double } \\
\text { work, representing the same product with different } \\
\text { tools in parallel } \rightarrow \text { lacking efficiency } \\
\end{array}$ \\
\hline Team & lacking communication & $\begin{array}{l}\text { no regular communication about current product ideas } \\
\text { and prototypes; lacking exploitation of prototyping as } \\
\text { a communication tool }\end{array}$ \\
\hline Organizational & $\begin{array}{l}\text { lacking appreciation and } \\
\text { resources for prototyping }\end{array}$ & $\begin{array}{l}\text { UX research and testing is not considered as a central } \\
\text { quality/business factor ("no time for that", not taken } \\
\text { seriously, especially low fidelity prototyping methods) }\end{array}$ \\
\hline Organizational & $\begin{array}{l}\text { lacking integration of } \\
\text { prototyping in development } \\
\text { processes }\end{array}$ & $\begin{array}{l}\text { role confusion about who is responsible for what, } \\
\text { inefficient/double work }\end{array}$ \\
\hline Organizational & $\begin{array}{l}\text { lacking support of appropriate } \\
\text { evaluation studies }\end{array}$ & $\begin{array}{l}\text { lacking access to relevant target groups (e.g., end- } \\
\text { users, real customers), design decisions are based on } \\
\text { assumptions about users rather than actual } \\
\text { feedback/insights } \rightarrow \text { lacking exploitation of } \\
\text { prototyping as evaluation tool }\end{array}$ \\
\hline Organizational & $\begin{array}{l}\text { lacking communication of } \\
\text { existing possibilities }\end{array}$ & $\begin{array}{l}\text { employees are not aware of internal opportunities and } \\
\text { support for prototyping (e.g., maker space, prototyping } \\
\text { workshops) }\end{array}$ \\
\hline
\end{tabular}


Based on barriers listed in Table 1, we deduced prototyping requirements. We reformulated identified barriers into four positive goals central for a successful application of prototyping at Siemens - and probably other companies:

- Appreciation: Position prototyping in development processes, UX research and testing with actual target group as mandatory

- Practical support: Provide guidelines for a systematic use of prototyping (e.g., positioning in the double diamond, choosing the right method for particular stakeholders), extend consulting services of prototyping at Siemens (e.g., maker space) to not only providing support on how to apply tools (e.g. laser cutter) but also on which kind of prototypes could be helpful for specific questions

- Collaboration: Develop guidelines for the efficient use of different prototyping tools and possibly helpful ways to express design decisions, avoid double work, exploit prototypes as a medium of communication and collaboration

- Internal communication and continued training: Integrate prototyping and communication about prototyping into daily business, introduce forums for communication and exchange among colleagues, exchange about best practices, enhance and leverage synergies

\subsection{Summary and conclusion: A first model of prototyping maturity from two perspectives}

Our focus group on prototyping in practice revealed several interesting insights on the understanding of prototyping from an industrial perspective, different levels of raising awareness behind a systematic choice of prototyping tools and methods, as well as typical barriers and requirements to successful prototyping. Across all these issues, participants' statements either referred to the individual or the organizational level. Hence, prototyping maturity can be considered along two perspectives. First, on an individual level, referring to the single individual's competencies, and second, on an organizational level, capturing to what degree prototyping is manifested in the organizations' processes, roles, and finally cultural values. Figure 2 and figure 3 display these insights in form of generalized models. The different levels suggested in the models are no direct results of the focus group but rather represent a first attempt to make the two perspectives on prototyping maturity available beyond the present project context.

As figure 2 suggests, prototyping competencies of individual stakeholders can range from basic knowledge about methods and the general importance of prototyping up to reflecting on their prototyping purpose and position in the process and therefore choosing a suitable method within a given context and applying it validly (see figure 2). Second, we consider prototyping as an indicator for a culture of digital innovation within a company and its readiness to support this. While a company can (and should) raise prototyping maturity through building up prototyping knowledge of internal prototyping stakeholders and provide guidelines for a systematic use of prototyping (e.g., positioning in the double diamond, choosing the right method for particular stakeholders), the value of prototyping also needs to be reflected within a company's culture and values. Ideally, the organization should promote a culture of trust, experimentation (e.g. role play is not ridiculous, stories about failures are shared), and learning from prototypes (and failures). Figure 3 displays these two perspectives. 


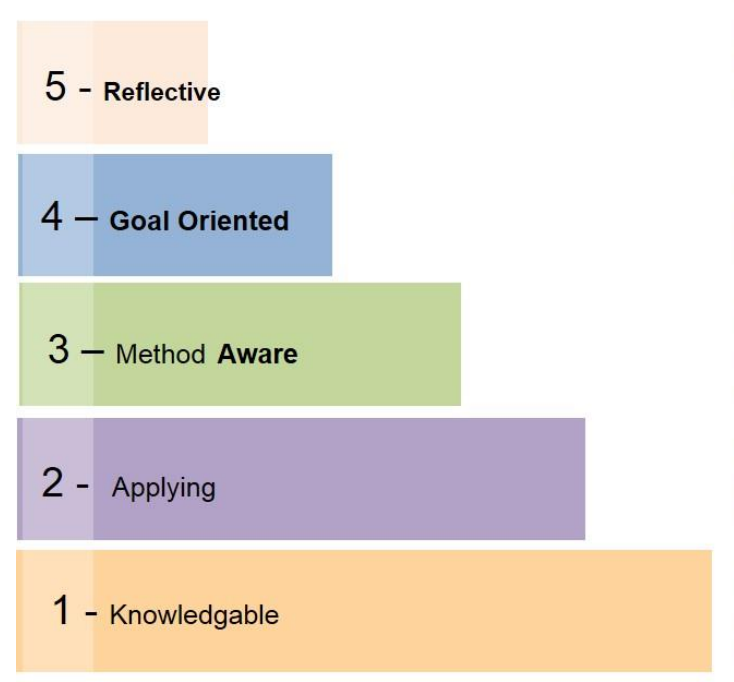

Level 5- people can deliberately reflect on their prototyping role, the position in the process and therefore choose an appropriate prototyping method in a given context and apply it in a valid way.

Level 4 - people know the challenges of prototyping; they are aware of differences in efficiency and validity of prototypes, depending on the purpose; they can phrase the purpose/focus of their prototype.

Level 3 - people are aware that there are different prototyping methods which are more or less appropriate depending on the purpose

Level 2 - people can practically apply and follow the procedure of dedicated prototyping methods (but no reflection on appropriateness)

Level 1 - people know that there are different prototyping methods and that prototyping is an important part of product development

Figure 2. Prototyping maturity levels of individual stakeholders (own graphic)

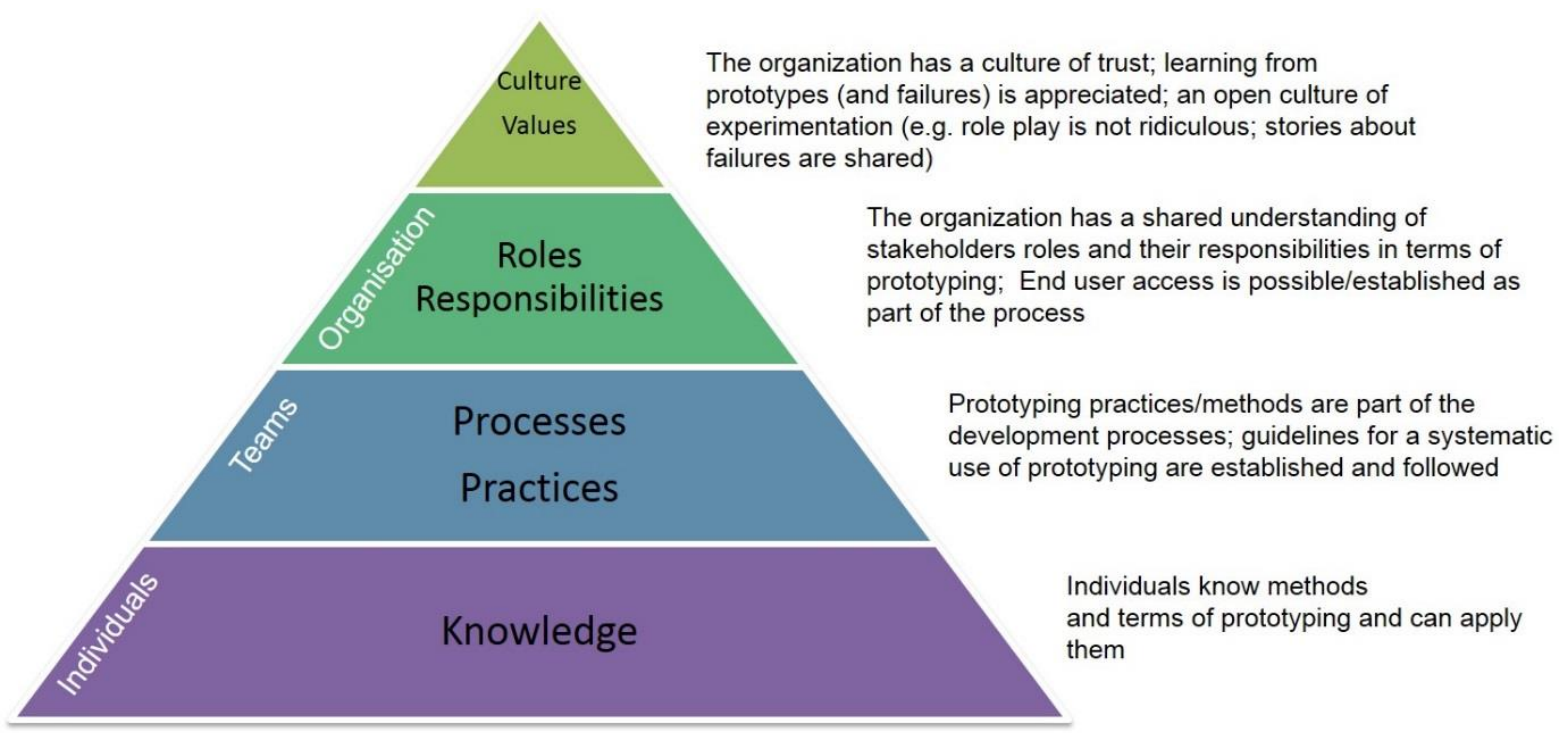

Figure 3. Prototyping maturity levels within an organization - from an individual to a cultural level (own graphic)

\section{REFLECTION FROM A COMPANY INTERNAL PERSPECTIVE}

As our industrial partner Siemens verbalized when reflecting about the results of the focus group, "these findings highlight many of the problems as we have experienced them in the application of design thinking in more than 40 projects in the last seven years". Obviously, there is a considerable gap between the practice of design thinking projects at a b2b industry player like Siemens and the understanding and ideal usage of prototyping as described in the literature or university applications of Design Thinking such as, for example, Stanford me310/SUGAR, the d.school courses as well as projects at the Aalto Design Factory. The focus group findings in combination with the double diamond perspective make the reasons for this gap and unused potential more comprehensible. In industrial contexts, there is often a lack of awareness of the variety of possibilities on how prototypes can be used and the value of these different usages at certain project stages. In general, prototypes in b2b development departments are often limited to usage in the solution space. Often, these usages come in two different granularities, initially as a descriptive and sometimes visual representation of the idea in a power point slide deck for approval and development support and later as a functional demonstrator to validate the idea's feasibility. On the contrary, the usage of prototypes to explore the 
so-called problem space, and to deepen the understanding of user needs and context requirements within the project is less common. Missing awareness can also be seen in the explicit application of prototypes in the sense of "build to think" - the usage of tangible artefacts to enact and explore the use case.

Besides lacking awareness of the different possibilities to profit from prototypes, another reason for their limited usage lays in short-term time management. Development teams within Siemens - like with many industry players - are facing pressure to become lean and agile, to provide explicit customer value in a very effective and efficient way. To save time and resources, many teams are immediately generating a high-resolution prototype (coding software) to address assumed customer/user needs. Diverging low resolution prototypes (paper scribbles of UIs) for early customer/user testing are often not even considered. That this routine could lead to overall "waste" due to later timely and costly design adjustment is theoretically known - but hard to reflect in concrete business numbers, and thus often practically neglected.

Finally, many development teams are lacking skills and competences, as well as access to material and tools for a broader use of prototyping beyond the present application. Siemens makes several offerings to address this. For example, the Design Thinking group within Siemens Corporate Technology is offering workshops to generate awareness, build up knowledge and foster skills in the selection, development and usage of various prototype forms and maturity levels. Additionally, in the last years, maker spaces have been built up in various locations. However, access restrictions to these facilities and resources for trained specialists for hands-on support are limiting the use of these offers and thus limit prototyping competencies among Siemens employees on a broad level.

\section{IMPLICATIONS FOR PROTOTYPING IN PRACTICE AND FUTURE RESEARCH}

The present focus group captured prototyping practices at one company within a limited sample of employees. This, of course, limits our findings. Further research in other organizations and with larger samples is necessary to check to what degree the present insights are generalizable, or what additional issues might occur. However, in order to provide basis for helpful next steps, we summarized the identified barriers to take full advantage of the power of prototyping in the following preliminary implications for prototyping in practice and future research:

- Organizational integration: Prototyping should be integrated in the companies' organizational and procedural design. Its application within product development should be prioritized in consideration of the projects character. Necessary resources should be assigned.

- Identify business impact: Decision makers have/should to be aware of the benefits of the various prototypes and that this value has business impact. As decision makers often require proof points, more research validating the value of different forms of prototypes in the problem as well as solution space is needed to bridge awareness to interest, desire and, finally, real action (in line with the so-called AIDA principle).

- Effective tools and processes: For effective and efficient prototyping, companies should select the most suitable tools for each application area. Lean application approaches should be developed and integrated into to development process to keep the effort low and leverage the benefit.

- Knowledge and skills: Finally, on the operational level, stakeholders who are involved in the development process have to build up knowledge, skills and competences to select and apply various prototyping tools. Therefore, training offers are needed and communities of experts that support other peer-groups internally in a non-bureaucratic manner could be highly useful.

\section{REFERENCES}

Blomkvist, J. and Holmlid, S. (2011). "Existing prototyping perspectives: considerations for service design". Nordes, Vol. 4.

Cash, P., Hicks, B. and Culley, S. (2015), “Activity Theory as a means for multi-scale analysis of the engineering design process: A protocol study of design in practice”, Design Studies, Vol. 38, pp. 1-32. http://dx.doi.org/10.1016/j.destud.2015.02.001 
Chakrabarti, A., Shea, K., Stone, R., Cagan, J., Campbell, M.I., Hernandez, N.V. and Wood, K.L. (2011), "Computer-Based Design Synthesis Research: An Overview", Journal of Computing and Information Science in Engineering, Vol. 11 No. 2, p. 021003. http://doi.org/10.1115/1.3593409

Chang, Y. N., Lim, Y. K. and Stolterman, E. (2008). "Personas: from theory to practices". In Proceedings of the 5th Nordic conderence on Human-computer interaction: building bridges, pp. 439-442. ACM.

Christoforakos, L. and Diefenbach, S. (2017). "Erfolgreiches Prototyping im Ideenstadium der Produktentwicklung”. In: Hess, S. and Fischer, H. (Eds.), Mensch und Computer 2017 - Usability Professionals 2017 (pp. 329-335). Gesellschaft für Informatik e.V, Regensburg. http://doi.org/10.18420/muc2017-up-0193.

Christoforakos, L., Diefenbach, S., Kohler, K. and Tretter, S. (2018). "Effektives Prototyping: Eine Stakeholderorientierte Perspektive". In: Hess, S. and Fischer, H. (Eds.), Mensch und Computer 2018 - Usability Professionals 2018 (pp. 103-113). Gesellschaft für Informatik e.V, Dresden.

Design Council (2007). "Eleven lessons: managing design in eleven global brands". Desk research report. https://www.designcouncil.org.uk/sites/default/files/asset/document/ElevenLessons_DeskResearchReport_ 0.pdf

Diefenbach, S., Chien, W.-C., Lenz, E. and Hassenzahl, M. (2013). "Prototypen auf dem Prüfstand”. Bedeutsamkeit der Repräsentationsform im Rahmen der Konzeptevaluation. i-com. Zeitschrift für interaktive und kooperative Medien, Vol. 12 No. 1, pp. 53-63.

Houde, S. and Hill, C. (1997). "What do prototypes prototype". In M. Helander, T. Landauer, and P. Prabhu (Eds.) Handbook of Human-Computer Interaction (2nd Ed.), pp. 367-381. Elsevier, Amsterdam.

Lim, Y., Stolterman, E. and Tenenberg, J. (2008). "The anatomy of prototypes: Prototypes as filters, prototypes as manifestations of design ideas”. ACM Transactions on Computer-Human Interaction, Vol. 15 No. 2.

Maier, J.R.A. and Fadel, G.M. (2009a), "Affordance based design: A relational theory for design", Research in Engineering Design, Vol. 20 No. 1, pp. 13-27. http://doi.org/10.1007/s00163-008-0060-3

Maier, J.R.A. and Fadel, G.M. (2009b), "Affordance-based design methods for innovative design, redesign and reverse engineering”, Research in Engineering Design, Vol. 20 No. 4, pp. 225-239. http://doi.org/10.1007/s00163-009-0064-7

Pahl, G. and Beitz, W. (1996), Engineering Design: A Systematic Approach, Springer, Berlin. http://dx.doi.org/10.1007/978-1-4471-3581-4

Pruitt, J. and Grudin, J. (2003, June). "Personas: practice and theory". In Proceedings of the 2003 conference on Designing for user experiences, pp. 1-15. ACM.

Schneider, K. (1996, May). "Prototypes as assets, not toys: why and how to extract knowledge from prototypes". In Proceedings of the 18th international conference on Software engineering, pp. 522-531. IEEE Computer Society.

\section{ACKNOWLEDGMENTS}

The researchers would like to thank the User Experience Design Group within Siemens Corporate Technology as well as the participants of the focus group for supporting this research. Part of this research has been funded by the German Federal Ministry of Education and Research (BMBF), project ProFI (FKZ: 01IS16015) and the Siemens AG, project Prototyping Readiness. 